[52] I.Saito, R. Nagata, T. Matsuura,Tetrahedron Lett. 1981,22,4231;J.Am.Chem.Soc. 1985, 107,6329.

[53] A. G. Kepka, L. I. Grossweiner, Photochem Photobiol. 1971, 14 ,621; ibid. 1973, 18, 49; W. Poppe, L. 1. Grossweiner, ibid. 1975, 22, 217.

[54] K. K. Rohatgi-Mukherjee, A. K. Gupta, Chem Phys. Lett. 1977, 46, 368; Photochem. Photobiol. $1978,27,539$.

[55] G. Braathen, P.-T. Chou, H. Frei, J. Phys. Chem. 1988, 92, 6610.

[56] W. L. Jolly, 'Modern Inorganic Chemistry', McGraw-Hill, New York, 1984, p. 181.

[57] M. Eigen, K. Kustin, J. Am. Chem. Soc. 1962, 84, 1355.

[58] A. J. Bard, R. Parsons, J. Jordan, 'Standard Potentials in Aqueous Solution', Marcel Dekker, New York, 1985.
[59] H. Gerischer, F. Willig, in 'Topics in Current Chemistry $61^{\prime}$, Ed. F. Boschke, Springer Verlag, Berlin, 1976, p. 31; T. Watanabe, A Fujishima, K Honda, in 'Energy Resources through Photochemistry and Catalysis', Ed. M. Grätzel, Academic Press, New York, 1983, p. 359; R. Memming, Progr. Surface Sci. 1984, 17, 7.

[60] J.A. Turner, J. Manassen, A. J. Nozik, Appl.Phys. Lett. 1980, 37, 488; D. Laser, A. J. Bard, J. Phys. Chem. 1976, 80, 459 .

[61] M.Grätzel, H. Frei,J.Phys.Chem. 1989,93, 7037.

[62] A. J. Nozik, Annu. Rev. Phys. Chem. 1978, 29, 189

[63] N. Vlachopoulos, P. Liska, J. Augustynski, M. Grätzel, J. Am. Chem. Soc. 1988, II0, 1216, and ref. cit. therein.

[64] P. Liska, N. Vlachopoulos, M. K. Nazeeruddin, P.

Compte, M. Grätzel,J.Am. Chem. Soc. 1988, 110. 3686; P. Compte, M. K. Nazeeruddin, F. P. Rotzinger, A.J.Frank, M.Grätzel,J.Mol.Catal.1989, 52,63 .

[65] D. J. Fitzmaurice, H. Frei, Langmuir 1991, $7, \cdots$.

[66] A. Henglein, Ber. Bunsenges. Phys. Chem. 1982, 86. 241; J. Moser, M. Grätzel, Hels. Chim. Acta 1982, 65, 1436.

[67] J. Desilvestro, M. Grätzel, L. Kavan, J. Moser, J. Am. Chem. Soc. 1985, 107, 2988.

[68] H. Frei, D. J. Fitzmaurice, M. Grätzel, Langmuir 1990, 6, 198.

[69] Conference Report by J. Haggin, C\&ENew's Aug. 31 1987, 18; Sept. 3 1990, 30; R. A. Sheldon, J. K. Kochi, 'Metal-Catalyzed Oxidations of Organic Compounds, Academic Press', New York, 1981.

\title{
Allocution prononcée à l'occasion de la remise du Prix Latsis 1990
}

\author{
le 19 novembre 1990 au Rathaus de Berne
}

\section{Geoffrey Bodenhausen*}

Monsieur le Conseiller fédéral,

Mesdames, Messieurs,

Chers arnis,

Contrairement à mes habitudes, je vous ai préparé un texte écrit, afin de partager avec vous quelques réflexions. J'ai une fois eu le plaisir d'écouter le très éloquent physico-chimiste Peter Atkins qui commencait son discours ainsi: 'If you think that I am reading from a piece of paper, well, that's precisely what I shall be doing'.

C'est d'abord une source de grande satisfaction de savoir que ce Prix n'est pas d'office destiné à un chimiste. En effet, cela vaut bien plus qu'une récompense attribuée par une association professionnelle à l'un de ses membres. Un prix de chimiste signalerait tout au plus que l'on jouit de la confiance de quelques collègues immédiats. Or ce Prix Latsis est d'une autre catégorie, et je m'en réjouis. Entendez-moi bien: je ne me fais pas d'illusions. Je me rends parfaitement compte qu'il est bien difficile - voire impossible - de comparer les mérites d'un mathématicien, d'un ingénieur ou d'un physico-chimiste. Je pense qu'il y a d'autres candidats en Suisse dont le mérite est au moins égal au mien, et je ne doute pas qu'il y ait un élément d'arbitraire - et partant d'injustice - dans le choix d'un candidat.

Cependant, je suis heureux de voir ce prix attribué à un chimiste. Je pense d'abord

\footnotetext{
*Correspondance: Prof. G. Bodenhausen Section de Chimie

Université de Lausanne

Rue de la Barre 2

$\mathrm{CH}-1005$ Lausanne
}

à la chimie au sens large, qu'elle soit analytique ou synthétique, qu'elle soit propre ou polluante, qu'elle soit traditionnelle ou qu'elle se situe à la lisière de spécialités modernes telles que la biologie moléculaire. Cette chimie représente un domaine extraordinairement vaste, d'une richesse indescriptible, qui est le théâtre d'un foisonnement d'idées d'une grande variété. Je crois que cette richesse est tout à fait inconnue du grand public.

Songez donc par exemple: quelle incroyable latitude s'offre à un enseignant universitaire lorsqu'il établit le programme d'un cours de chimie! Pour les uns, la pratique de la chimie est un métier essentiellement manuel, un artisanat au sens noble du terme, qui requiert avant tout de l'adresse et de la débrouillardise. Pour d'autres (et je me situe plutôt parmi ceux-là), cette même chimie est une activité plutôt intellectuelle, qui se pratique un crayon à la main, à la rigueur à l'aide d'un ordinateur. J'avoue que je n'ai plus tenu d'éprouvette dans les mains depuis plus de quinze ans. Est-ce à dire que je suis un renégat, un chimiste qui s'est subrepticement recyclé en physicien? Point du tout! Je crois que, malgré la fâcheuse tendance au morcellement, la chimie demeure une science essentiellement indivisible.

Si je prends la défense de la chimie aujourd'hui, c'est parce que je la sens mal aimée et incomprise. La presse ne parle que pollution, elle ne s'intéresse à la chimie que s'il y a un accident à déplorer, elle se borne trop souvent à refléter la fascination bien humaine (et un brin morbide) devant le dan-

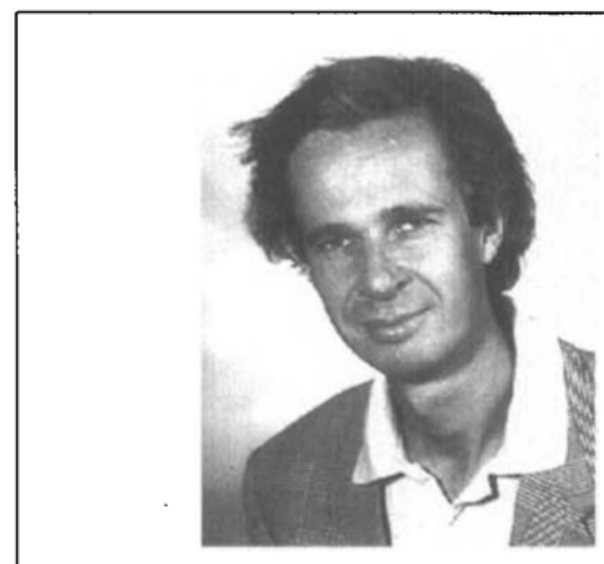

Geoffiey Bodenhausen: né le 7 mai 1951 à La Haye, Pays-Bas. Ecole primaire à La Haye, Pays-Bas. Ecole Internationale de Genève, 1963-1970 (Maturité fédérale type C). Ecole polytechnique fédérale de Zürich, 1970-1974(Diplôme de chimie). Thèse de doctorat sous la direction du Prof. Ray Freeman, Laboratoire de chimie physique, Universitéd'Oxford, 1975-77 (D.Phil.). Scholarship of the Salter's Company, London, 1976-77. University of California at San Diego, 1978: Recherches en spectroscopie à multiples quanta (Prof. R.L.Vold et R.R. Vold'). Member of Research Staff, Francis Bitter National Magnet Laboratory, Massachusetts Institute of Technology, 1979-1980: Recherches en résonance magnétique des substances solides. Assistant, puis maître-assistant, Laboratoire de chimie physique, Ecole polytechnique fédérale de Zürich. 1980-1985: Enseignement en chimie physique, rédaction d'une monographie et recherches variées en spectroscopie. Prix 1983 de l'Association des chimistes suisses. Privat-docent en chimie physique, Ecole polytechnique fédérale de Zürich, 1984-88. Professeur associé, Institut de chimic organique, Universitć de Lausanne, $1985-$ présent. Directeur, Institut de chimie organique, sept. 1987-déc. 1990. Prix Latsis 1990 décerné par le Fonds national de la recherche scientifique.

ger. A lire certains articles, on serait parfois amené à croire que les chimistes sont personnellement responsables du fait que la combustion du pétrole dégage du gaz carbonique!

Alexandre Vialatte a dit (à propos de la langue française et de son orthographe): "Quand on est amoureux de sa langue, on l'aime dans ses difficultés. On l'aime telle quelle, comme sa grand 'mère, avec ses rides et ses verrues.» C'est dans ce sens que je vois la chimie, que je revendique mon appartenance à cette communauté scientifi- 
[52] I.Saito, R. Nagata, T. Matsuura,Tetrahedron Lett. 1981,22,4231;J.Am.Chem.Soc. 1985, 107,6329.

[53] A. G. Kepka, L. I. Grossweiner, Photochem Photobiol. 1971, 14 ,621; ibid. 1973, 18, 49; W. Poppe, L. 1. Grossweiner, ibid. 1975, 22, 217.

[54] K. K. Rohatgi-Mukherjee, A. K. Gupta, Chem Phys. Lett. 1977, 46, 368; Photochem. Photobiol. $1978,27,539$.

[55] G. Braathen, P.-T. Chou, H. Frei, J. Phys. Chem. 1988, 92, 6610.

[56] W. L. Jolly, 'Modern Inorganic Chemistry', McGraw-Hill, New York, 1984, p. 181.

[57] M. Eigen, K. Kustin, J. Am. Chem. Soc. 1962, 84, 1355.

[58] A. J. Bard, R. Parsons, J. Jordan, 'Standard Potentials in Aqueous Solution', Marcel Dekker, New York, 1985.
[59] H. Gerischer, F. Willig, in 'Topics in Current Chemistry $61^{\prime}$, Ed. F. Boschke, Springer Verlag, Berlin, 1976, p. 31; T. Watanabe, A Fujishima, K Honda, in 'Energy Resources through Photochemistry and Catalysis', Ed. M. Grätzel, Academic Press, New York, 1983, p. 359; R. Memming, Progr. Surface Sci. 1984, 17, 7.

[60] J.A. Turner, J. Manassen, A. J. Nozik, Appl.Phys. Lett. 1980, 37, 488; D. Laser, A. J. Bard, J. Phys. Chem. 1976, 80, 459 .

[61] M.Grätzel, H. Frei,J.Phys.Chem. 1989,93, 7037.

[62] A. J. Nozik, Annu. Rev. Phys. Chem. 1978, 29, 189

[63] N. Vlachopoulos, P. Liska, J. Augustynski, M. Grätzel, J. Am. Chem. Soc. 1988, II0, 1216, and ref. cit. therein.

[64] P. Liska, N. Vlachopoulos, M. K. Nazeeruddin, P.

Compte, M. Grätzel,J.Am. Chem. Soc. 1988, 110. 3686; P. Compte, M. K. Nazeeruddin, F. P. Rotzinger, A.J.Frank, M.Grätzel,J.Mol.Catal.1989, 52,63 .

[65] D. J. Fitzmaurice, H. Frei, Langmuir 1991, $7, \cdots$.

[66] A. Henglein, Ber. Bunsenges. Phys. Chem. 1982, 86. 241; J. Moser, M. Grätzel, Hels. Chim. Acta 1982, 65, 1436.

[67] J. Desilvestro, M. Grätzel, L. Kavan, J. Moser, J. Am. Chem. Soc. 1985, 107, 2988.

[68] H. Frei, D. J. Fitzmaurice, M. Grätzel, Langmuir 1990, 6, 198.

[69] Conference Report by J. Haggin, C\&ENew's Aug. 31 1987, 18; Sept. 3 1990, 30; R. A. Sheldon, J. K. Kochi, 'Metal-Catalyzed Oxidations of Organic Compounds, Academic Press', New York, 1981.

\title{
Allocution prononcée à l'occasion de la remise du Prix Latsis 1990
}

\author{
le 19 novembre 1990 au Rathaus de Berne
}

\section{Geoffrey Bodenhausen*}

Monsieur le Conseiller fédéral,

Mesdames, Messieurs,

Chers arnis,

Contrairement à mes habitudes, je vous ai préparé un texte écrit, afin de partager avec vous quelques réflexions. J'ai une fois eu le plaisir d'écouter le très éloquent physico-chimiste Peter Atkins qui commencait son discours ainsi: 'If you think that I am reading from a piece of paper, well, that's precisely what I shall be doing'.

C'est d'abord une source de grande satisfaction de savoir que ce Prix n'est pas d'office destiné à un chimiste. En effet, cela vaut bien plus qu'une récompense attribuée par une association professionnelle à l'un de ses membres. Un prix de chimiste signalerait tout au plus que l'on jouit de la confiance de quelques collègues immédiats. Or ce Prix Latsis est d'une autre catégorie, et je m'en réjouis. Entendez-moi bien: je ne me fais pas d'illusions. Je me rends parfaitement compte qu'il est bien difficile - voire impossible - de comparer les mérites d'un mathématicien, d'un ingénieur ou d'un physico-chimiste. Je pense qu'il y a d'autres candidats en Suisse dont le mérite est au moins égal au mien, et je ne doute pas qu'il y ait un élément d'arbitraire - et partant d'injustice - dans le choix d'un candidat.

Cependant, je suis heureux de voir ce prix attribué à un chimiste. Je pense d'abord

\footnotetext{
*Correspondance: Prof. G. Bodenhausen Section de Chimie

Université de Lausanne

Rue de la Barre 2

$\mathrm{CH}-1005$ Lausanne
}

à la chimie au sens large, qu'elle soit analytique ou synthétique, qu'elle soit propre ou polluante, qu'elle soit traditionnelle ou qu'elle se situe à la lisière de spécialités modernes telles que la biologie moléculaire. Cette chimie représente un domaine extraordinairement vaste, d'une richesse indescriptible, qui est le théâtre d'un foisonnement d'idées d'une grande variété. Je crois que cette richesse est tout à fait inconnue du grand public.

Songez donc par exemple: quelle incroyable latitude s'offre à un enseignant universitaire lorsqu'il établit le programme d'un cours de chimie! Pour les uns, la pratique de la chimie est un métier essentiellement manuel, un artisanat au sens noble du terme, qui requiert avant tout de l'adresse et de la débrouillardise. Pour d'autres (et je me situe plutôt parmi ceux-là), cette même chimie est une activité plutôt intellectuelle, qui se pratique un crayon à la main, à la rigueur à l'aide d'un ordinateur. J'avoue que je n'ai plus tenu d'éprouvette dans les mains depuis plus de quinze ans. Est-ce à dire que je suis un renégat, un chimiste qui s'est subrepticement recyclé en physicien? Point du tout! Je crois que, malgré la fâcheuse tendance au morcellement, la chimie demeure une science essentiellement indivisible.

Si je prends la défense de la chimie aujourd'hui, c'est parce que je la sens mal aimée et incomprise. La presse ne parle que pollution, elle ne s'intéresse à la chimie que s'il y a un accident à déplorer, elle se borne trop souvent à refléter la fascination bien humaine (et un brin morbide) devant le dan-

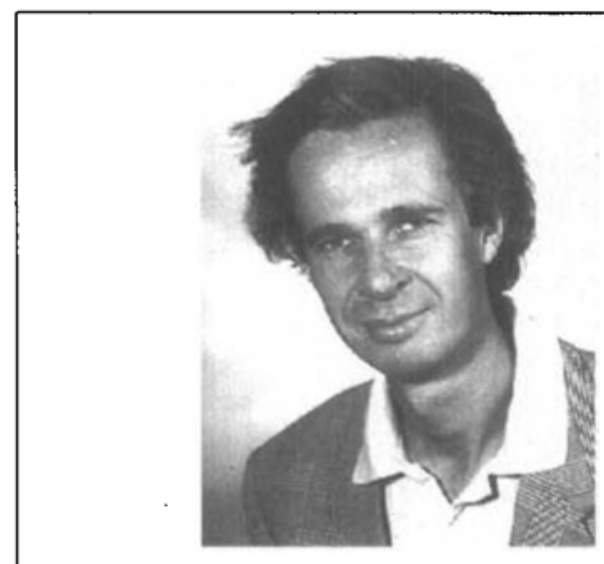

Geoffiey Bodenhausen: né le 7 mai 1951 à La Haye, Pays-Bas. Ecole primaire à La Haye, Pays-Bas. Ecole Internationale de Genève, 1963-1970 (Maturité fédérale type C). Ecole polytechnique fédérale de Zürich, 1970-1974(Diplôme de chimie). Thèse de doctorat sous la direction du Prof. Ray Freeman, Laboratoire de chimie physique, Universitéd'Oxford, 1975-77 (D.Phil.). Scholarship of the Salter's Company, London, 1976-77. University of California at San Diego, 1978: Recherches en spectroscopie à multiples quanta (Prof. R.L.Vold et R.R. Vold'). Member of Research Staff, Francis Bitter National Magnet Laboratory, Massachusetts Institute of Technology, 1979-1980: Recherches en résonance magnétique des substances solides. Assistant, puis maître-assistant, Laboratoire de chimie physique, Ecole polytechnique fédérale de Zürich. 1980-1985: Enseignement en chimie physique, rédaction d'une monographie et recherches variées en spectroscopie. Prix 1983 de l'Association des chimistes suisses. Privat-docent en chimie physique, Ecole polytechnique fédérale de Zürich, 1984-88. Professeur associé, Institut de chimic organique, Universitć de Lausanne, $1985-$ présent. Directeur, Institut de chimie organique, sept. 1987-déc. 1990. Prix Latsis 1990 décerné par le Fonds national de la recherche scientifique.

ger. A lire certains articles, on serait parfois amené à croire que les chimistes sont personnellement responsables du fait que la combustion du pétrole dégage du gaz carbonique!

Alexandre Vialatte a dit (à propos de la langue française et de son orthographe): "Quand on est amoureux de sa langue, on l'aime dans ses difficultés. On l'aime telle quelle, comme sa grand 'mère, avec ses rides et ses verrues.» C'est dans ce sens que je vois la chimie, que je revendique mon appartenance à cette communauté scientifi- 
que. Je vois la chimie non pas comme quelque maîtresse envoûtante, mais comme un être vivant, imparfait, qui a ses laideurs, mais aussi ses beautés.

Considérant la désolante cote de popularité de la chimie, je crois que le temps est venu de lancer une contre-attaque. Il faut expliquer aux jeunes qu'il y a là un domaine d'une grande richesse et une activité professionnelle passionnante.

La Suisse offre une infrastructure exceptionnelle à la pratique de la chimie. A Lausanne, nous espérons emménager dans quelques années dans un nouveau bâtiment à Dorigny. Nous sommes décidés à construire une petite merveille de fonctionnalité, un espace réellement privilégié, un rêve de laboratoire en somme. Nous avons confiance que l'on nous offrira les moyens de le faire, car nous nous réjouissons de l'appui généreux du Canton de Vaud et de la Confédération. Nous avons confiance aussi que nous saurons y conduire des travaux dignes de cet environnement! Cependant: il y a une ombre au tableau, dont j'hésite à parler, car bien de mes collègues préfèrent la passer sous silence. C'est que la chimie attire de moins en moins d'étudiants. C'est un phénomène qui touche l'ensemble de la Suisse. Déjà les associations professionnelles tirent la sonnette d'alarme. L'industrie engage actuellement des chimistes étrangers à tour de bras: plus de $60 \%$ de ceux qui ont récemment été engagés ont fait leurs études à l'étranger. La «production» indigène des docteurs ès sciences chimiques, d'une centaine par an pour toute la Suisse, ne suffit meme pas à satisfaire la moitié de la demande... Si vous me permettez une remarque un peu provocatrice, nous allons vers un régime du chercheur-saisonnier. Je ne suis pas sûr que ce soit là un bon pari.

Il y a un autre développement qui m'inquiète. De plus en plus, nous voyons des jeunes filles qui s'engagent dans les études de chimie. Je suis le premier à m'en réjouir, car rien ne justifierait que ce métier soit réservé aux hommes. Cependant, je $m$ 'inquiète lorsque la proportion féminine monte à $80 \%$ des étudiants, voire au-delà. Dans la société qui est la nôtre, cela n'est pas un bon signe: pensez aux métiers presque entièrement réservés aux femmes, tel celui des infirmières. Je ne puis m'empêcher de penser que leur statut social (et leur salaire) sont bien trop modestes par rapport à leur mérite. Au risque de vous choquer, je perçois dans ce double développement, c'est-à-dire la féminisation excessive et l'importation massive de main-d'œuvre étrangère, une tendance à la prolétarisation.

Il faut absolument que la chimie suisse les universités et hautes écoles, les départements cantonaux de l'instruction publique, mais aussi l'industrie et les associations professionnelles - développe une campagne de relations publiques, une opération de charme, afin de réconcilier l'opinion publique avec la chimie.

Ce samedi soir, j'ai vu un «spot» publici- taire à la télévision qui était destiné à faciliter le recrutement d'apprentis-mécaniciens. Ce spot, parailleurs fort séduisant, était accompagné du commentaire surprenant: «les métiers de l'automobile sont utiles à l' environnement $\gg$. Je vous demande: si le lobby des garagistes et des importateurs d'automobiles est capabled'une telle opération de charme, pourquoi la chimie suisse, qui défend des enjeux bien plus importants, en serait-elle incapable?

Je vous donnerai un autre exemple de l'excessive retenue, je dirais même de la pudeur quelque peu effarouchée de notre industrie chimique. La Suisse s'est dotée d'un instrument parfaitement adapté pour que la Confédération puisse apporter un soutien à des projets de recherche impliquant des partenaires industriels et universitaires. Je veux parler de la Commission pour l'encouragement de la recherche scientifique (la CERS, peut-être plus connue sous son sigle allemand de KWF). Les crédits de cette institution jouent un rôle important pour notre groupe de recherche à l'Université de Lausanne, et nos partenaires industriels, le groupe Bruker-Spectrospin, ont bien compris l'enjeu, sans doute parce qu'il s'agit d'une entreprise jeune et dynamique qui est à la pointe du développement des instruments de chimie analytique. Mais lorsque mes collègues, engagés, eux, dans une chimie préparative et synthétique, s'adressent à leurs partenaires habituels, qui sont les grandes maisons de la chimie suisse, ils se heurtent le plus souvent à une résistance insurmontable. Toute allusion à un financement mixte entre la Confédération et l'industrie privée (carc'est bien de cela qu'il s'agit) suffit pour effaroucher les dirigeants de ces entreprises, car ils préfèrent s'en tenir à une politique ultra-libérale qui exclut tout financement de l'Etat, un point de vue à mon sens par trop dogmatique et certainement contraire à l'esprit d'ouverture dont nous avons besoin.

Le métier de chimiste devrait attirer plus de monde, pourvu qu'il soit mieux connu. C'est en Suisse, paradoxalement, que la «Verfremdung» entre le grand public et la chimie a pris les proportions les plus inquiétantes. Dans les pays de l'espace européen qui nous entourent, le divorce semble bien moins marqué. C'est l'une des raisons pour les quelles nous, chimistes universitaires, souhaitons jouer la carte européenne à fond. Et je suis fier de vous dire, en ma capacité de directeur de l'un des instituts de chimie de l'Université de Lausanne, que nous avons d'ores et déjà pris le taureau par les cornes, que nous pratiquons déjà une politique d'ouverture tous azimuts. A défaut de statistiques qui portent sur l'ensemble de l'institut, je me contenterai de vous indiquer les nationalités réprésentées dans notre groupe de recherche, en intégrant sur une période de cinq ans: nous avons accueilli, pour des périodes allant de six mois à cinq ans, 3 anglais, 3 allemands, 2 italiens, 2 autrichiens, 2 suédois, une yougoslave de
Slovénie, un roumain, et, pour bonne mesure, 11 suisses. Je vous assure que ce groupe bigarré est le lieu de débats passionnants!

Au niveau de notre institut, nous nous efforçons de dépoussiérer les structures, d'insuffler une vie nouvelle, de multiplier les centres d'initiative et de décision, et surtout - maître-mot, s'il en est - de faire confiance à ceux qui sont plus jeunes que nous, afin qu'ils puissent à leur tour s'épanouir sans entraves. Je souhaite que nous ne soyons pas les seuls à suivre une telle politique!

Il y a tant à faire. Les sciences moléculaires sont en pleine expansion, la biologie moléculaire passe d'une victoire à l'autre, la chimie analytique parvient de plus en plus à sonder les secrets de la matière, la chimie préparative est le théâtre d'un «design moléculaire» où tout, ou presque, semble permis, une architecture microscopique étonnante, où la créativité artistique peut s'exprimer en inventant des structures qui sont, au sens fort du terme, fantastiques.

J'ai retrouvé récemment, dans le grenier de la maison où j'ai passé mon adolescence, une collection de dessins qui datent de l'époque où je voulais devenir architecte. Je sens que le métier de l'architecte et celui du chimiste sont très proches. C'est simplement l'échelle de l'objet sur lequel on travaille qui diffère. Mais à part cela, nous, chimistes, pensons, comme les architectes, en termes d'espaces, volumes et de structures.

Je comprends mal la dichotomie que l'on veut souvent faire, déjà dès les classes prégymnasiales, entre les scientifiques et les littéraires. Je crois que c'est une erreur profonde. De toute façon, l'activité professionnelle d'un scientifique l'amène avant tout à manier la langue: discuter et convaincre, rédiger des articles, et même faire des discours!

Revenons les pieds sur terre, l'espace d'un moment, si vous le voulez bien. Le prix Latsis nous a été accordé pour nos travaux en résonance magnétique nucléaire. C'est une méthode interdisciplinaire par excellence, puisqu'elle est utilisée avec succès aussi bien pour permettre aux médecins de «regarder» à l'intérieur du corps humain que pour déterminer la structure des molécules. Grâce aux techniques que nous avons aidé à développer, nous obtenons des «images» des molécules. Ces images demandent à être décodées, car ce ne sont pas de simples photographies. Les images sur lesquelles nous travaillons ressemblent à des paysages parcourus de collines, de crêtes montagneuses, jalonnés de pics vertigineux. (Il n'est pas surprenant que ces méthodes jouissent d'une grande popularité en Suisse.) La hauteur du relief nous renseigne, entre autres choses, sur les distances qui séparent les noyaux au sein des molécules, distances qui sont infimes, à savoir de l'ordre du millionième de millimètre. L'interprétation de ces images peut être faite «manuellement» par des chimistes, physiciens, biologistes 
moléculaires et autres experts chevronnés L'originalité de nos recherches consiste en ce que nous essayons de transférer à un ordinateur une partie de ce travail fastidieux. Comme l'ordinateur ne possède pas l'intelligence d'un expert - loin s'en faut - il s'agit de définir une stratégie appropriée, afin que la machine puisse «reconnaître» des motifs caractéristiques dans les images, et proposer un modèle de la molécule. L'intérêt de cette recherche réside en partie dans le fait qu'elle n'aboutira sans doute jamais parfaitement, car l'intuition humaine du chercheur, son imagination et sa créativité s'avèrent irremplaçables.

Le laudatio que vous venez d'entendre mentionne aussi une méthode nouvelle qui permet de visualiser des réactions chimiques qui se produisent lorsque les molécules s"entrechoquent en phase gazeuse. La technique nouvelle, que nous avons baptisée «résonance cyclotronique à deux dimensions» est née de l'application à des ions, c'est-à-dire à des fragments chargés de molécules, d'une méthode de mesure conçue à l'origine pour étudier le magnétisme nucléaire. C'est un très bon exemple de «fertilisation croisée» entre deux domaines de recherche relativement éloignés, que nous avons pu mener à bien grâce à la proximité à Lausanne de notre Université et de l'Ecole polytechnique fédérale.

Il y a eu d'autres travaux issus de notre groupe qui auraient pu être mentionnés: nos travaux théoriques sur l'imagerie médicale, les techniques qui permettent aux physiologistes de distinguer le sodium intra- et extracellulaire, les études de relaxation qui permettront peut-être un jour de mieux déterminer la forme tri-dimensionelle des molécules, et les nombreuses techniques destinées à obtenir des images de plus haute résolution et de meilleure qualité.

Malgré tous nos efforts d'éviter la spécialisation à outrance, notre programme de recherche peut paraître bien mince, trop étroitement confiné dans une spécialité ésotérique. En promenant un regard critique sur ce qu'il est convenu d'appeler mon «œuvre», je me demande: c'est donc cela, une carrière scientifique qui satisfait aux normes en vigueur? N'est-ce pas un abus que de vous faire croire qu'il s'agit là de travaux de valeur? N'ai-je pas simplement obnubilé le jugement du Fonds national par une véritable avalanche de publications, plutôt que par leur qualité et leur original ité? $Q u$ 'ai-je donc bien pu faire de répréhensible pour vous induire ainsi en erreur?

Je trouve quelques éléments de réponse à ces questions en lisant «Betrayers of the Truths de William Broad et Nicholas Wade, que mon collègue et ami Pierre Feschotte $m$ 'a fait lire en traduction francaise, intituléc «La souris truquée» [1]. Bien que ce livre soit quelque peu superficiel, les auteurs ont le mérite de poser quelques questions très dures à la communauté des chercheurs. SeIon leur analyse, le milieu scientifique est corrompu, infesté de gens malhonnêtes qui falsifient leurs résultats ou qui fabriquent de toutes pièces des rapports sur des expériences qu'ilsn'ont jamais faites. Si je vous parle de cela aujourd'hui, c'est précisément parce que les auteurs soupçonnent que l'espoir de se voir attribuer de prestigieuses récompenses puisse fomenter une ambiance où l'ambition l'emporte sur la quête désintéressée de la vérité. Vous voyez que la question est d'actualité.

Au vu de ces virulentes accusations, je plaide coupable sur deux plans: celui de m'être abandonné - non sans quelque volupté - à ce que le sociologue Robert Merton a appellé «l' effet Matthieu» et celui de n'avoir pas toujours su résister aux vertiges de «l'illusion».

Qu'est-ce donc que l'effet Matthieu? Il s'agit du phénomène complexe «de détournement de paternité» du travail scientifique. Car il faut bien admettre que ce n'est pas un effort solitaire qui m'a propulsé sur ce podium. C'est d'ailleurs pourquoi j'ai demandé à la Fondation Latsis d'inviter à cette cérémonie l'ensemble des collaborateurs, anciens et actuels, de notre groupe àl'Université de Lausanne. Cen'est pas un simple geste de politesse, mais bien le souci de reconnaître une dette incontestable: sans eux, je ne me trouverais pas ici. Je me suis souvent interrogé quant à la légitimité de m'arroger la propriété intellectuelle de ce qui ne m'appartient pas. Il faut bien admettre que les directeurs de thèse et chefs de groupe de recherche sont investis d'un pouvoir considérable vis-à-vis de leurs collaborateurs, un pouvoir qui est sans doute excessif et manque de garde-fous. Merton a parlé de l'effet Matthieu par allusion à l'évangile de saint Matthieu, où il est écrit: «Car on donnera à celui qui a, mais à celui qui n'a pas, on ôtera même ce qu'il a'. (Ceux d'entre vous qui me connaissent personnellement seront sans doute un peu surpris de m'entendre citer un texte biblique: c'est que l'ignorance de ces textes me permet de les découvrir dans toute leur fraîcheur...) Et si aujourd 'hui «on donne à celui qui a», ce n'est pas à l'argent que je pense, mais à ce qu'en français un peu vieilli on appellerait la gloire. C'est surtout en ce sens que je suis aujourd'hui dans la position d'un débiteur vis-à-vis de mes collaborateurs. Si vous croyez que je pèche par excès d'humilité, prenez donc cet exemple: l'analyse informatisée de spectres, dont il est question dans le laudatio, se fait grâce à des logiciels très complexes qui ont été développés dans notre laboratoire. Or, je suis entièrement tributaire de mes assistants, car je suis tout à fait incapable d'écrire moimême le moindre programme d'ordinateur... $\mathrm{Ma}$ fonction dans ce développement s'est bornée à poser des questions. De là, à s'en approprier les résultats, il y a un pas que Socrate n'aurait peut-être pas franchi.

Je me sens aussi constamment débordé par le zèle de mes collaborateurs sur le plan théorique, c'est-à-dire sur le plan où un directeur de thèse devrait normalement garder une avance confortable... Tout ceci me place dans une position vulnérable. Je ne crois pas qu'il y a des recettes pour redresser la situation. Je crois par contre que les questions de responsabilité qui sont soulevées ici transcendent la pratique scientifique. C'est pourquoi j'ai aussi demandé à la fondation Latsis d'inviter l'un de mes maîtres de l'époque où je fréquentais l'Ecole Internationale de Genève, non seulement parce qu'il m'a fait connaître la chimie (ce qui est peu de chose en somme), mais surtout parce qu'il m'a fait comprendre très tôt que la pratique scientifique ne devait en aucun cas être séparée d'une approche humaniste.

Je vous ai parlé de l'effet Matthieu et des illusions. Qu'est-ce qu'un scientifique peut bien entendre par illusion? Je me contenterai d'un exemple plutôt que de vous donner une définition fastidieuse. Vous connaissez sans doute l'abbé Gregor Mendel, considéré comme le père de la génétique et peut-être même de toute la biologie moderne. Il avait une idée à la fois simple et forte de l'hérédité. Ses expériences sur les pois lisses et ridés devaient étayer sa théorie. Un petit texte anonyme, publié en 1972 dans Horticultural Science (et cité par Broad et Wade [1]), nous révèle toute la problématique des expériences de Mendel:

«Au commencement était Mendel, ruminant ses pensées solitaires. Puis il dit: «Qu'il y ait des pois», et il y eut des pois, et cela était bon. Puis il mit ces pois dans le jardin et leur dit: «Croissez et multipliez, différenciez-vous et assortisse-vous indépendamment.» Ainsi firent-ils, et cela était bon. Puis advint que Mendel rassembla ses pois et les sépara en graines lisses et ridées; il appela les lisses dominantes, ef les ridées récessives, etcela était bon. Mais Mendel vit alors qu' il y avait 450 pois lisses et 102 pois ridés. Celan'était pas bon. Car la loi stipule qu' il doit y aloir trois lisses pour un ridé. Et Mendel se dit en lui-même: "Gott im Himmel, c'est là l'auvre d'un ennemi qui aura semé des mauvais pois dans mon jardin à la faveur de la nuit». Et Mendel, pris d' un juste courroux, frappa sur la table et dit: «Eloignez-vous de moi, pois maudits et diaboliques, retournez dans les ténèbres où vous serez dévorés par les rats et les souris!' Et il enfut ainsi: il ne resta plus que 300 pois lisses et 100 pois ridés, et cela était bon. Excellent même. Et Mendel le publia.» Le drame, c'est que ces statistiques sont trop exactes pour être vraies. Malgré tout notre respect pour l'œuvre de Mendel, force est de constater qu'il a essayé de nous mystifier.

Si je vous cite ce texte, ce n'est pas seulement pour vous faire sourire, mais parce que cette histoire me donne une forte impression de «déjà vu». Toutes proportions gardées, j'ai commis des impostures semblables à celles de Mendel. Et - au risque de vous scandaliser - j'affirme que je n'en ai pas honte.

Le domaine de notre spécialisation se réjouit d'une base théorique extrêmement solide. Nous pouvons tout calculer, ou presque tout, et souvent nous savons d'avance 
quels résultats nos expériences devraient donner. Bref, nous sommes dans une situation assez semblable à l'abbé Gregor Mendel, qui, lui aussi, a dû avoir une foi inébranlable en sa propre théorie. Nous sommes donc souvent tentés d'élaguer des résultats qui ne nous conviennent pas. Cette situation $m$ 'inquiète, même si je crois que nous sommes mûs par un esprit honnête. Non, mon inquiétude vient de ce que notre comportement s'inscrit en porte-à-faux par rapport au mode de travail idéalisé que le grand public prête aux scientifiques. Nous sommes à mille lieues d'une recherche impersonnelle, objective, et dénuée de passion.

Ces lectures ont réellement semé un doute dans mon esprit. J'ai trouvé quelque réconfort chez Albert Einstein, qui a écrit, dans une lettre à Heisenberg: «C'est la théoriequi décide c'e que nous pouvons observer». C'est bien ce que Mendel a fait, et c'est bien ce que nous faisons tous les jours.

Si Einstein ne constitue pas une référence suffisante, voici un passage de Sigmund Freud que mon épouse m'a montré hier [2]: "Wir haben oftmals die Forderung vertreten gehört, dass eine Wissenschaft über klaren und scharf definierten Grundbegriffen aufgehaut sein soll. In Wirklichkeit beginnt keine Wissenschaft mit solchen Definitionen, auch die exakteste nicht. Der richtige Anfang der wissenschaftlichen Tätigkeit besteht vielmehr in der Beschreibung von Erscheinungen, die dann weiterhin gruppiert. angeordnet und in Zusammenhänge eingetragen werden. Schon bei der Beschreibung kann man es nicht vermeiden, gewisse ab- strakte Ideen auf das Material anzuw'enden, die man irgendwoher, gewiss nicht aus der neuen Erfahrung allein, herbeiholt.»

Vous voyez que Freud et Einstein tiennent essentiellement le même langage. Vous voyez aussi qu'il y a une tension, pour ne pas dire une contradiction, sur des questions très fondamentales. Qu'est-ce que tout cela peut bien avoir à faire à nos travaux? Et bien, je soutiens que c'est dans cette tension que réside tout l'intérêt de la recherche, et qu'elle prend des formes particulièrement fécondes dans le domaine de la chimie moderne.

Pour finir, quelques mots à propos du prix Latsis. Vous savez que ce prix n'est pas purement honorifique, encore que, jusqu'à présent, seuls quelques journalistes en quête de sensations fortes ont voulu savoir ce que nous entendions faire d'une si belle somme. Eh bien, sachez que jusqu'ici, nous nous sommes bornés à acheter l'œuvre complète de Freud, dont vous venez d'entendre un extrait. D'autres folies suivront! Mais audelà de ces petits fantasmes, il y a surtout le sens d'une grande liberté. La liberté de rêver, mais aussi celle de s'engager dans certains débats. Fortifié d'une autorité nouvelle, je me sens en droit d'exprimer une opinion sur certains sujets d'actualité. En politique de la science, certes, mais au-delà, je crois qu'il faut s'engager sur des grands débats comme celui de l'énergie nucléaire. Non nécessairement pour plaider qu'on y renonce purement et simplement, mais surtout pour apporter une touche modératrice dans un débat par trop passionné. Pour éviter que l'on peigne le diable sur la muraille, mais aussi pour modérer les partisans qui font souvent preuve d'une confiance trop naïve en la technologie. J'ai été souvent tenté de m'engager dans ce débat (par exemple en adressant une lettre de lecteur à un grand quotidien), maisj'ai toujours craint de n'être pas pris au sérieux. Eh bien, aujourd hui je crois que quelques-uns au moins m'écouteront. C'est surtout de cette force nouvelle que je suis reconnaissant. Reconnaissant au Conseil du Fonds national, et reconnaissant à la Fondation Latsis.

Monsieur le Conseiller fédéral, Mesdames et Messieurs, cher amis, je vous remercie de votre attention.

Reçu le 14 décémbre 1990

[1] W. Broad, N. Wade, "Betrayers of the truth". Simon and Schuster, New York, 1982, "La souris truquée», trad. C. Jeanmougin. Seuil, Paris, 1987.

[2] S. Freud, "Triebe und Tricbschicksale", 1915 (Studienausgabe S. Fischer Verlag. Frankfurt. Band III, S. 81). Voici la traduction française par J. Laplanche et J.-B. Pontalis (dans «Mctapsychologie», Gallimard, Paris, p. 11): «Nous an'oms son'ent entendu formuler levigence smirante: whe scionce doit être construite sur des's come'pts fondamentaux chairs et netteme'nt difinis. En réalitó, ancume science. mômo la plus cisacte., ne conmence par de lelles définitions. Le víritable'

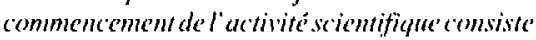
plurôr dans la description de phéneminess, yui

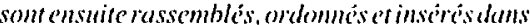
des relations. Dans la description. dijai, an ne peat éviter d'appliquer all materiel iertaines idées abstraites que' l'on puise ici ou lì c't erertuinement pas dans la sealc expériene' acturelle',n

Chimia 45 (1991) 193-195

(C) Schweiz. Chemiker-Verband: ISSN 0009-4293

\title{
Der neue Entwurf des Schweizerischen Umweltschutzgesetzes
}

\author{
Georg Karlaganis*
}

\section{Die schweizerische Umweltschutzge- setzgebung}

Ausser dem Umweltschutzgesetz(USG) befassen sich noch zahlreiche andere Bundesgesetze mit den Anliegen des Umweltschutzes. Zur Zeit der Gründung der Berner

*Korrespondenz: PD Dr. Georg Karlaganis Bundesamt für Umwelt, Wald und Landschaft Hallwylstrasse 4 $\mathrm{CH}-3003$ Bern
Chemischen Gesellschaft vor 100 Jahren existierte allerdings erst das Fabrikgesetz/ Arbeitsgesetz (1877/1964). Es folgten das Forstpolizeigesetz (1902), das Gewässerschutzgesetz (1955/1971), das Natur- und Heimatschutzgesetz (1966), das Fischereigesetz (1973) und das Raumplanungsgesetz (1979).

Im Jahre 1971 ist unsere Bundesverfassung mit einem Umweltschutz-Artikel (Art. $24^{\text {scpics }}$ ) ergänzt worden. Dieser Verfassungsauftrag führte zu unserem Unweltschutzgesetz, welches seit dem 1. Januar 1985 in Kraft ist. Der vom Verfassungsartikel anvi- sierte Schutz gilt dem Menschen und seiner natürlichen Umwelt. Schutzobjekt sind neben der menschlichen Gesundheit, welche das psychische Wohlbefinden einschliesst, auch die Umweltelemente selbst als Grundlage allen Lebens.

Der Zweckartikel des Umweltschutzgesetzes gibt dem wie folgt Ausdruck: «Dieses Gesetz soll Menschen, Tiere und Pflanzen, ihre Lebensgemeinschaften und Lebensräume gegen schädliche oder lästige Einwirkungen schützen und die Fruchtbarkcit des Bodens erhalten» (Art. 1, Absatz 1).

Das Umweltschutzgesetz basiert auf mehreren wichtigen Grundprinzipien: dem Vorsorgeprinzip, dem Prinzip der Bekämpfung an der Quelle, dem Verursacherprinzip, dem Kooperationsprinzip und dem Prinzip der ganzheitlichen Betrachtungsweise.

Das Umweltschutzgesetz regelt die Bereiche Luftreinhaltung, Lärmbekämpfung, Abfälle, umweltgefährdende Stoffe und Schutz des Bodens. Das Unweltschutzgesetz ist ein Rahmengesetz. Die Konkretisierung erfolgte durch eine Reihe von Verordnungen: Luftreinhalte-Verordnung (LRV, 1986), Lärmschutz-Vcrordnung (LSV. 